\title{
Theoretical Analysis of Selective Relaying, Cooperative Multi-Hop Networks with Fairness Constraints
}

\author{
Erwu Liu ${ }^{\dagger}$, Qinqing Zhang ${ }^{\ddagger}$ and Kin K. Leung ${ }^{\dagger}$ \\ ${ }^{\dagger}$ Department of Electrical and Electronic Engineering, Imperial College, London, UK \\ Email:\{erwu.liu, kin.leung\}@imperial.ac.uk \\ ${ }^{\ddagger}$ Applied Physics Laboratory, Johns Hopkins University, USA \\ ${ }^{\ddagger}$ Department of Computer Science, Johns Hopkins University, USA \\ Email:qinqing.zhang@jhuapl.edu
}

\begin{abstract}
We consider the problem of selective relaying in multi-hop networks. At each slot, a relay and a node along the optimal non-cooperative path are opportunistically selected to transmit to the next-hop node in a cooperative manner. Being a promising scheme for fair resource allocation, the proportional fair scheduling (PFS) algorithm provides excellent balance between throughput and fairness via multi-user diversity and game-theoretic equilibrium. To maximize the overall utility along a cooperative multi-hop path, we apply the proportional fair (PF) criterion in selecting nodes and relays for cooperative transmission. Furthermore, we analyze and provide an analytical expression for end-to-end throughput of an opportunistic relaying, cooperative multi-hop path with proportional fairness constraints over a Rayleigh flat-fading channel. To our knowledge, it is the first time that a closed-form expression is obtained for the throughput of a proportional fair relaying, cooperative multi-hop path. This research is an extension of previous theoretical work on PF for cellular networks.
\end{abstract}

\section{INTRODUCTION}

Being a new form of spatial diversity, cooperative diversity [1]-[3] can achieve full diversity in the number of cooperative nodes. Several relay protocols are proposed in [3]. According to their forwarding strategy, relays can be classified as amplifyand-forward, decode-and-forward and compress-and-forward. An amplify-and-forward relay acts as an analog repeater by retransmitting an amplified version of the received signal. Instead, a decode-and-forward relay attempts to decode, regenerates and retransmits an exact copy of the original signal. A compress-and-forward relay node quantizes the received signal from the source, encodes the samples into a new packet and retransmits it to the destination. Throughout, we assume the decode-and-forward relay strategy.

In a multi-hop network with $C$ relays at each hop, the maximum diversity gain provided by the cooperation among relays is $C$-fold regardless of the number of hops [4]. To achieve the full spatial diversity order, selective relaying, i.e., only the best relay is selected for forwarding the signal at each

This work was supported, in part, by Johns Hopkins University, Applied Physics Laboratory's internal research and development funds. hop, is a good candidate which requires minimum cooperation among the relays and which can be performed in a distributed way [5]. In selective relaying, opportunistic policy is used to maximize the throughput, i.e., the relay with the highest combined signal-to-noise ratio $(S N R)$ is selected at each hop. It is known that this greedy strategy results in highly unfair resource utilization and eventually, it will violate the quality of service (QoS) requirements of those overly-used relays when they have their own service to serve. Obviously, the performance degradation due to opportunistic relaying is even worse in multi-hop networks as the number of the overly-used relays is significantly increased. Hence, one has to be more careful when considering the trade-off between efficiency and fairness in selective relaying, multi-hop networks.

In the considerable efforts to deal with the trade-off between optimal system throughput and fairness, the proportional fair scheduling (PFS) algorithm is proposed [6]. By exploiting multi-user diversity and game-theoretic equilibrium, the PFS algorithm provides excellent balance between throughput and fairness (see [7]-[9] and the references therein). In light of this, this paper proposes an opportunistic relaying algorithm with proportional fairness constraints for the considered multi-hop network. Built upon previous work on PFS [8], we then analyze the end-to-end throughput performance of a proportional fair relaying (PFR), multi-hop path. Specifically, we derive the closed-form expression for end-to-end throughput of a PFR multi-hop path. To the best of our knowledge, it is the first time that a closed-form expression is obtained for end-to-end throughput in such networks.

The paper is structured as follows. In Section II, we first describe the assumptions made in our study of the problem and the resulting problem formulations, we then propose the PFR algorithm that maximizes the overall utility of a multihop relay path. After that, analytical results which extend our prior work on proportional fairness $(\mathrm{PF})$ are presented in Section III. Finally, in Section IV, we present simulation results to validate the theoretical findings, followed by the conclusion in Section V. 


\section{System ModeL}

We assume a relay setting where there are several relays and one source-destination pair for each hop along a multihop path. Both relays and nodes are equipped with only a single antenna. As shown in Fig. 1, along an $m$-hop path $<$ $n_{1}, n_{2}, \ldots, n_{m}, d>,\left|\Theta_{i}\right|$ relays are clustered as relay set $\Theta_{i}$ at the $i$-th hop. We assume non-overlapped relay sets, i.e., $\Theta_{i} \cap \Theta_{j}=\varnothing, \forall i \neq j$. TDMA is used so that only one hop $i$ is active at each slot.

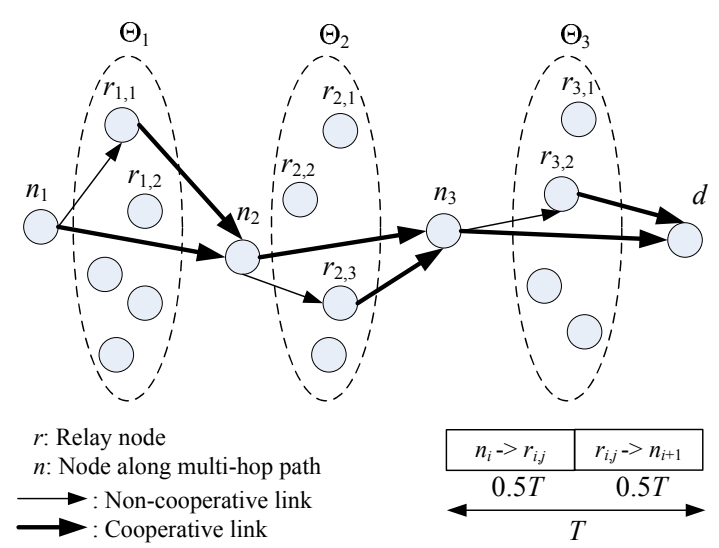

Fig. 1. Selective relaying over a cooperative multi-hop path

For a multi-hop path in Fig. 1, selective decode-and-forward relaying is assumed. i.e., at each hop $i$, only one relay relay $r_{i, j} \in \Theta_{i}$ is selected for forwarding the packet. We adopt socalled 3-node relaying as the cooperative transmission method at each hop. That is, once a relay $r_{i, j}$ is selected, node $n_{i}$ will first transmit to $r_{i, j}$ in the first half slot, $n_{i}$ and $r_{i, j}$ will then cooperatively transmit to the next-hop node $n_{i+1}$ in the second half slot. We use $n_{i} \rightarrow r_{i, j},\left(n_{i}, r_{i, j}\right) \rightarrow n_{i+1}$ to denote the non-cooperative link and the cooperative link, respectively. A non-cooperative link and the accompanying cooperative link together compose a 3-node relay link. Obviously, there are $\left|\Theta_{i}\right|$ 3-node relay links at the $i$-th hop.

Unless otherwise specified, bandwidth is normalized to $1 \mathrm{~Hz}$. Let $R_{i, j}^{N C}[t+1], R_{i, j}^{C}[t+1]$ be the instantaneous capacities of the non-cooperative link $n_{i} \rightarrow r_{i, j}$ and the cooperative link $\left(n_{i}, r_{i, j}\right) \rightarrow n_{i+1}$, and $R_{i, j}[t+1]$ be the instantaneous capacity of the corresponding 3-node relay link, in next time slot $t+1$. $R_{i, j}[t]$ 's $k$-point moving average throughput up to time $t$ is denoted by $\mu_{i, j ; k}[t]$. In opportunistic relaying without fairness constraints, the objective is to maximize the overall throughput $\sum_{i, j} \mu_{i, j ; k}[t]$ of all 3-node relay links. Instead, in opportunistic relaying with proportional fairness constraints, the objective is to maximize the overall utility of all 3-node relay links. This is formulated as follows

$$
\max \sum_{i=1}^{N} \sum_{j=1}^{\left|\Theta_{i}\right|} \ln \left(\mu_{i, j ; k}(t)\right) .
$$

s.t.

$$
\begin{gathered}
I_{i, j}[t+1]=\left\{\begin{array}{rr}
1 & \text { if } n_{i} \text { and } r_{i, j} \text { are scheduled for } \\
\text { cooperative transmission. } & \text { elser }
\end{array}\right. \\
\sum_{i=1}^{N} \sum_{j=1}^{\Theta_{i}} I_{i, j}[t+1]=1 . \\
\mu_{i, j ; k}[t+1]=\left(1-\frac{1}{k}\right) \mu_{i, j ; k}[t]+I_{i, j}[t+1] \times \frac{R_{i, j}[t+1]}{k} . \\
R_{i, j}[t+1]=\frac{1}{2} \times \min \left(R_{i, j}^{N C}[t+1], R_{i, j}^{C}[t+1]\right) .
\end{gathered}
$$

(2)and (3) indicate that at each time slot, only one relay and one accompanying node will be selected to cooperatively transmit to the next-hop node at next time slot.

The factor $\frac{1}{2}$ in (5) accounts for the two-phase transmission, i.e., node $n_{i}$ first transmits to a relay $r_{i, j}$, then $n_{i}$ and $r_{i, j}$ cooperatively transmit to the next-hop node $n_{i+1}$.

For the above optimization problem, we propose the following algorithm.

\section{Proportional fair relaying (PFR) algorithm:}

1) Initially, determine relay set $\Theta_{i}$ for each hop $i$. Set initial throughput $\mu_{i, j ; k}[0]$. At each slot $t$,

2) Estimate the next slot capacities $R_{i, j}^{N C}[t+1], R_{i, j}^{C}[t+1]$ of the non-cooperative link $n_{i} \rightarrow r_{i, j}$ and the cooperative link $\left(n_{i}, r_{i, j}\right) \rightarrow n_{i+1}$, respectively.

3) Calculate $R_{i, j}[t+1]=\frac{1}{2} \min \left(R_{i, j}^{N C}[t+1], R_{i, j}^{C}[t+1]\right)$. Calculate PF metric $M_{i, j ; k}[t+1]=R_{i, j}[t+1] / \mu_{i, j ; k}[t]$.

4) $\left(i^{*}, j^{*}\right)=\arg \max _{(i, j)} M_{i, j ; k}[t+1]$ is selected to indicate that in the next time slot $t+1$, node $n_{i^{*}}$ will first transmit to relay $r_{i^{*}, j^{*}}$, then $n_{i^{*}}$ and $r_{i^{*}, j^{*}}$ will cooperatively transmit to the next-hop node $n_{i^{*}+1}$. Update $I_{i^{*}, j^{*}}[t+1]=1, I_{\forall i \neq i^{*}, j \neq j^{*}}[t+1]=0$

5) According to (4), update the throughput $\mu_{i, j ; k}[t+1]$ of each 3-node relay link.

6) Goto 2) to proceed to next slot.

By viewing each 3-node relay link in Fig. 1 as a user in a cellular network, the PFR algorithm above functions as a PFS algorithm used in cellular networks. It is well-known that PFS maximizes the overall utility $\sum_{i} \ln \left(\mu_{i}\right)$ where $\mu_{i}$ is the throughput of user $i$. Obviously, the PFR algorithm is the optimal solution of (1).

\section{ANALysis OF PFR OVER A RAYLEIGH FLAT-FADING CHANNEL}

In this section, we analyze the proposed PFR algorithm over Rayleigh flat-fading channels, and provide closed-form theoretical results. For the analysis, we use the following assumptions:

- Both the non-cooperative link and the cooperative link are Rayleigh flat faded;

- The fading between any different links (no matter they are non-cooperative links or cooperative links) is independent but not necessarily identical distributed; 
- Channel fading keeps constant over each slot, and varies independently from slot to slot;

- The maximal ratio combiner (MRC) is used at each nexthop node to combine the signals from the last-hop node and the relay.

To include the distance-dependent path loss, we note that the instantaneous $S N R$ is the multiplication of the average value $\overline{S N R}$ (determined by path loss and shadowing) with the random fading variable. This results in a single random variable with mean that jointly models path loss and fading. Consequently, the instantaneous $S N R$ can be modeled as an exponentially distributed random variable with mean $\overline{S N R}$ determined by the distance-dependent path loss. This model has likewise been used in [3].

We use $S N R_{i, j}^{N C}, S N R_{i, j}^{C}$ to denote the instantaneous $S N R s$ of the non-cooperative link $n_{i} \rightarrow r_{i, j}$ and the cooperative link $\left(n_{i}, r_{i, j}\right) \rightarrow n_{i+1}$, respectively.

We use $\overline{S N R_{i, j}^{S R}}, \overline{S N R_{i, i+1}^{S D}}, \overline{S N R_{j, i+1}^{R D}}$ to denote the mean $S N R s$ of link $n_{i} \rightarrow r_{i, j}$, link $n_{i} \rightarrow n_{i+1}$, and link $r_{i, j} \rightarrow n_{i+1}$, respectively. Here $S R, S D, R D$ are used to indicate the transmission source relay, source destination and relay destination, respectively.

Due to Rayleigh fading, the instantaneous $S N R$ of a noncooperative link is an exponentially distributed random variable with a probability density function (pdf) given by

$$
\rho_{i, j}^{N C}(x)=\frac{1}{\overline{S N R_{i, j}^{S R}}} \times e^{-x / \overline{S N R_{i, j}^{S R}}} .
$$

In MRC, the combined SNR is the sum of the SNRs of each individual diversity branch. The pdf of the instantaneous $S N R$ of a cooperative link is then given by

$$
\rho_{i, j}^{C}(x)=\frac{1}{\overline{S N R_{i, i+1}^{S D}}+\overline{S N R_{j, i+1}^{R D}}} \times e^{-x /\left(\overline{S N R_{i, j}^{S D}}+\overline{S N R_{j, i+1}^{R D}}\right)} .
$$

We can then write the capacities of the non-cooperative link and the cooperative link as follows

$$
\begin{gathered}
R_{i, j}^{N C}=\log _{2}\left(1+S N R_{i, j}^{N C}\right) . \\
R_{i, j}^{C}=\log _{2}\left(1+S N R_{i, j}^{C}\right) .
\end{gathered}
$$

Substituting (8) and (9) into (5), we have

$$
\begin{aligned}
& R_{i, j}[t+1]=\frac{1}{2} \times \min \left(R_{i, j}^{N C}[t+1], R_{i, j}^{C}[t+1]\right) \\
& =\frac{1}{2} \times \log _{2}\left(1+\min \left(S N R_{i, j}^{N C}[t+1], S N R_{i, j}^{C}[t+1]\right)\right) \cdot(10)
\end{aligned}
$$

We know that the distribution of the minimum of multiple independently exponential random variables is also an exponential distributed random variable, i.e., for $n$ independently exponential distributed random variables $g_{1}, g_{2}, \ldots, g_{n}$ with parameters $\lambda_{1}, \lambda_{2}, \ldots, \lambda_{n}, g=\min \left\{g_{1}, g_{2}, \ldots, g_{n}\right\}$ is also exponentially distributed, with parameter

$$
\lambda=\lambda_{1}+\lambda_{2}+\ldots+\lambda_{n} .
$$

We then have the capacity of the 3-node relay link,

$$
R_{i, j}[t+1]=\frac{1}{2} \times \log _{2}\left(1+\operatorname{SNR}_{i, j}[t+1]\right) .
$$

where $S N R_{i, j}$ is an exponentially distributed random variable with a pdf given by

$$
\begin{aligned}
\rho_{i, j}(x)= & \left(\frac{1}{\overline{S N R_{i, i+1}^{S D}}+\overline{S N R_{j, i+1}^{R D}}}+\frac{1}{\overline{S N R_{i, j}^{S R}}}\right) \\
& \quad-\left(\frac{x}{\overline{S N R_{i, i+1}^{S D}}+\overline{S N R_{j, i+1}^{R D}}}+\frac{x}{\overline{S N R_{i, j}^{S R}}}\right) .
\end{aligned}
$$

(13) indicates that the mean value of $S N R_{i, j}$ is

$$
\overline{S N R_{i, j}}=\frac{1}{\left(\frac{1}{\overline{S N R_{i, i+1}^{S D}}+\overline{S N R_{j, i+1}^{R D}}}+\frac{1}{\overline{S N R_{i, j}^{S R}}}\right) .} .
$$

[10] and [11] have shown that, in Rayleigh or Ricean flatfading networks, the shannon capacity $R=\log _{2}[1+S N R]$ can be accurately approximated by Gaussian distribution. Specially, for a single-input-single-output (SISO) link over a Rayleigh flat-fading channel where $S N R$ is exponential distributed, the Gaussian distribution is characterized by a mean and variance given as follows

$$
\begin{gathered}
E[R]=\int_{0}^{\infty} e^{-x} \times \log _{2}(1+\overline{S N R} \times x) d x \\
\sigma^{2}=\int_{0}^{\infty} e^{-x} \times\left(\log _{2}(1+\overline{S N R} \times x)\right)^{2} d x-(E[R])^{2} .
\end{gathered}
$$

With (12)-(16), we obtain the Gaussian distribution that characterizes the capacity of the 3 -node relay link $\left\{n_{i} \rightarrow\right.$ $\left.r_{i, j},\left(n_{i}, r_{i, j}\right) \rightarrow n_{i+1}\right\}$

$$
\begin{aligned}
E\left[R_{i, j}\right] & =\frac{1}{2} \int_{0}^{\infty} e^{-x} d x \\
& \times \log _{2}\left(1+\frac{x}{\left(\frac{1}{\overline{S N R_{i, i+1}^{S D}}+\overline{S N R_{j, i+1}^{R D}}}+\frac{1}{\overline{S N R_{i, j}^{S R}}}\right)}\right) . \\
\sigma_{i, j}^{2}= & -\left(E\left[R_{i, j}\right]\right)^{2}+\frac{1}{4} \int_{0}^{\infty} e^{-x} d x \\
& \times\left(\log _{2}\left(1+\frac{1}{\left(\frac{1}{\overline{S N R_{i, i+1}^{S D}}+\overline{S N R_{j, i+1}^{R D}}}+\frac{1}{\overline{S N R_{i, j}^{S R}}}\right)}\right)\right)^{2} .
\end{aligned}
$$

According to our previous work on PFS [8], we have the following lemma:

Lemma 1 (PFS Throughput) For an N-user cellular network implementing the PFS algorithm, each user's average throughput is solely determined by its own channel statistics when the instantaneous capacity is Gaussian. In particular, the average throughput of user $i$ is given by

$$
\begin{aligned}
E\left[\mu_{i}\right] \approx & \frac{E\left[R_{i}\right]}{N} \times\left(1-\left[F_{(0,1)}\left(-M_{i}\right)\right]^{N}\right) \\
& +\int_{-M_{i}}^{\infty} y \sigma_{i} f_{(0,1)}(y) \times\left[F_{(0,1)}(y)\right]^{N-1} d y .
\end{aligned}
$$


where $R_{i}$ and $\mu_{i}$ are the instantaneous capacity and throughput of user $i, E\left[R_{i}\right]$ and $\sigma_{i}$ denote the statistical average and standard deviation of $R_{i}, M_{i}=E\left[R_{i}\right] / \mu_{i}, f_{(0,1)}(\cdot)$ and $F_{(0,1)}(\cdot)$ are the pdf and cdf of zero mean, unit variance standard normal distribution.

With (17), (18) and (19), we have the important theoretical result for this research.

Theorem 2 (PFR Throughput) For a 3-node relaying, cooperative m-hop path $\zeta$ over Rayleigh fading channels, the overall utility of all 3-node relay links is maximized when PFS is used to schedule a 3-node relay link at a time. Moreover, the average throughput $\gamma_{\zeta}$ of path $\zeta$ is given by

$E\left[\gamma_{\zeta}\right]=\min _{1 \leq i \leq m}\left(\begin{array}{c}\sum_{j=1}^{\left|\Theta_{i}\right|} E\left[R_{i, j}\right] / \sum_{j=1}^{m}\left|\Theta_{k}\right| \\ \times\left(1-\left[F_{(0,1)}\left(-M_{i, j}\right)\right]^{\sum_{k=1}^{m}\left|\Theta_{k}\right|}\right) \\ +\sum_{j=1}^{\left|\Theta_{i}\right|} \sigma_{i, j} \int_{-M_{i, j}}^{\infty} y f_{(0,1)}(y) d y \\ \times\left[F_{(0,1)}(y)\right]^{\left(\sum_{k=1}^{m}\left|\Theta_{k}\right|-1\right)}\end{array}\right)$.

where $E\left[R_{i, j}\right]$ and $\sigma_{i, j}$ are given by (17) and (18), $M_{i, j}=$ $E\left[R_{i, j}\right] / \sigma_{i, j}, \Theta_{i}$ is the relay set at the $i$-th hop and $\left|\Theta_{i}\right|$ is the number of relays in $\Theta_{i}$, respectively.

Proof: We can view the multi-hop path in Fig. 1 as a cellular network, and each 3-node relay link as an individual user in the cellular network. Obviously, there are $N=\sum_{k=1}^{m}\left|\Theta_{k}\right|$ "users" in the "cellular" network. Since the instantaneous capacity of a "user" is characterized by a statistically independent Gaussian distribution given by (17) and (18), according to Lemma 1, we have the average throughput $\tau_{i}$ of the $i$-th hop,

$$
\begin{aligned}
\tau_{i}= & \frac{\sum_{j=1}^{\left|\Theta_{i}\right|} E\left[R_{i, j}\right] \times\left(1-\left[F_{(0,1)}\left(-M_{i, j}\right)\right]^{\sum_{k=1}^{m}\left|\Theta_{k}\right|}\right)}{\sum_{j=1}^{m}\left|\Theta_{k}\right|} \\
& +\sum_{j=1}^{\left|\Theta_{i}\right|} \sigma_{i, j} \int_{-M_{i, j}}^{\infty} y f_{(0,1)}(y) \times\left[F_{(0,1)}(y)\right]^{\left(\sum_{k=1}^{m}\left|\Theta_{k}\right|-1\right)} d y .
\end{aligned}
$$

Given a path $\zeta$ in multi-hop network, it is well-known that end-to-end path throughput is determined by the minimum link throughput of the path [12], i.e.,

$$
E\left[\gamma_{\zeta}\right]=\min _{1 \leq i \leq m}\left(\tau_{i}\right) .
$$

We then have (20).

\section{Numerical and Simulation Results}

We first performed numerical experiments to evaluate the proposed PFR algorithm over a multi-hop path.

In numerical experiments, we use the following settings for the multi-hop path with relays: Each hop has the same number of relays, i.e., $\forall i=1 \sim m,\left|\Theta_{i}\right|=C ; \forall j=1 \sim C, \overline{S N R_{i, i+1}^{S D}}=$ $3 \mathrm{~dB}, \overline{S N R_{i, j}^{S R}}=12 \mathrm{~dB}, \overline{S N R_{j, i+1}^{R D}}=12 \mathrm{~dB}$;
For comparison purpose, we use two baseline models. In the first model, there is no relay at each hop and the hops (i.e., the links) along the multi-hop path are activated in an round-robin (RR) manner. In this baseline model, end-to-end throughput of an m-hop path can be readily expressed as

$$
E\left[\gamma_{\zeta}\right]=\min _{1 \leq i \leq m}\left(\frac{E\left[R_{i, i+1}\right]}{m}\right) .
$$

where the average throughput $E\left[R_{i, i+1}\right]$ of the $i$-th hop is determine by (15), i.e.,

$$
E\left[R_{i, i+1}\right]=\int_{0}^{\infty} e^{-x} \times \log _{2}\left(1+\overline{S N R_{i, i+1}^{S D}} \times x\right) d x .
$$

In the second model, there is no relay at each hop and the hops (i.e., the links) along the multi-hop path are activated in a PF manner. In this baseline model, end-to-end throughput of an m-hop path can be readily expressed as

$$
E\left[\gamma_{\zeta}\right]=\min _{1 \leq i \leq m}\left(\frac{E\left[R_{i, i+1}\right] \times\left(1-\left[F_{(0,1)}\left(-M_{i, i+1}\right)\right]^{m}\right)}{m}+\sigma_{i, i+1}\right) .
$$

where $E\left[R_{i, i+1}\right], \sigma_{i, i+1}$ are determine by (15) and (16).

Fig. 2 depicts the numerical results comparing end-to-end throughput of the proposed PFR multi-hop and the baseline models. As expected, the proposed PFR multi-hop outperforms the RR multi-hop and the PF multi-hop.

For reference, we plot in Fig. 3 the throughput gain of PFR multi-hop over RR multi-hop, i.e., $\left.E\left[\gamma_{\zeta}\right]\right|_{(\mathrm{PFR})} /\left.E\left[\gamma_{\zeta}\right]\right|_{(\mathrm{RR})}$. Obviously, the throughput gain is typically $1.5 \sim 2.5$.

Next we conduct simulations to validate the theoretical results. Simulation parameters are: 8000 time slots, moving average factor $k=500$. Refer to Fig. 1, nodes and relays are placed in an area of $\left.7 \times 12 \mathrm{~km}^{2} .<n_{1}, n_{2}, n_{3}, d\right\rangle$ is the optimal non-cooperative path for the communication between $n_{1}$ and $d .\left|\Theta_{1}\right|=5,\left|\Theta_{2}\right|=4,\left|\Theta_{3}\right|=4$. The received average $S N R$ is given by $S N R_{d_{0}}-10 \alpha \log _{10}\left[d / r_{0}\right]$, where $\alpha$ is path loss exponent (typically $2 \sim 5$ ), $d_{0}=500 \mathrm{~m}$ is the reference distance, $S N R_{d_{0}}=35 \mathrm{~dB}$ is the reference $S N R$ at $d_{0}$. Nodes/relays use SISO for communications. We assume

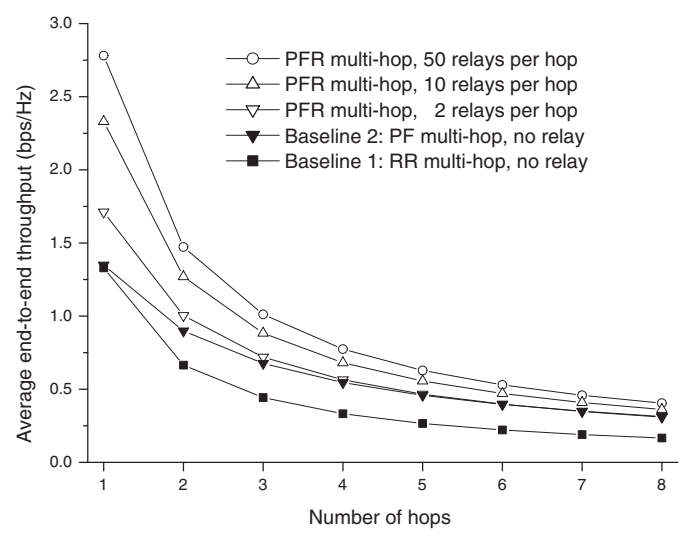

Fig. 2. End-to-end throughput: PFR multi-hop vs baseline models 


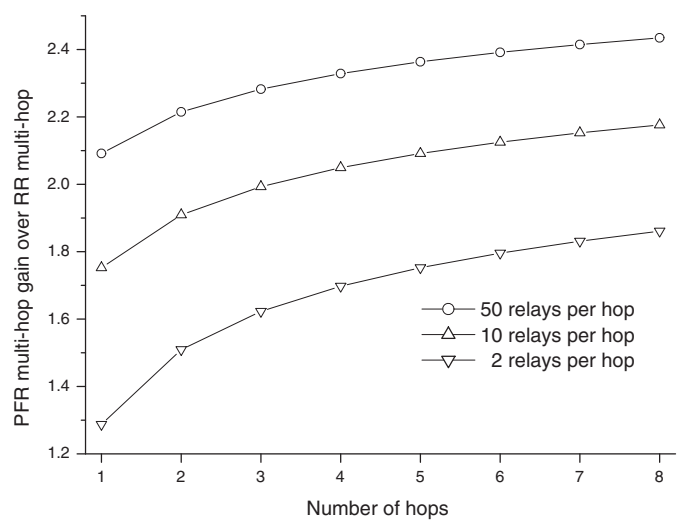

Fig. 3. Throughput gain of PFR multi-hop over RR multi-hop

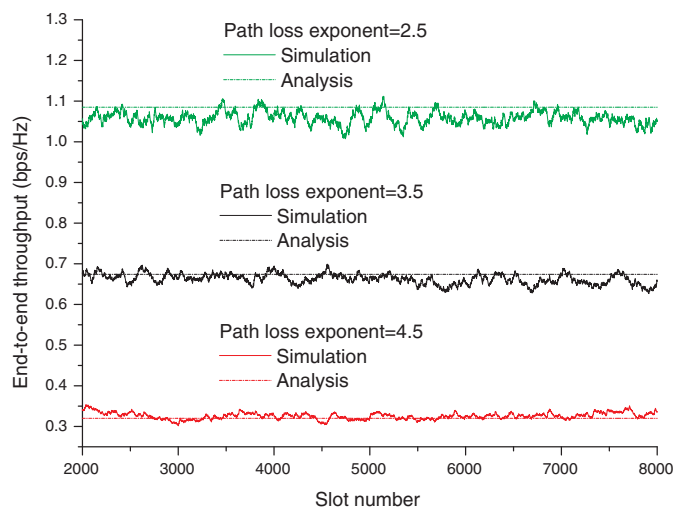

Fig. 4. End-to-end throughput: Simulation vs Analysis

Rayleigh fading. The validity of our theoretical results is illustrated in Fig. 4 where the dot lines are for the average end-to-end throughput from analysis.

\section{CONCLUSION}

Selective relaying is a promising cooperative method to achieve the full spatial diversity order. There are two selective relaying methods: Pure opportunistic relaying without fairness constraints and opportunistic relaying with fairness constraints. Pure opportunistic relaying without fairness constraints introduces serious QoS issues for overly-used relays in multi-hop networks. To facilitate researches on efficient and fair multihop networks, we proposed and analyzed a cooperative multihop network in the presence of selective relays and with proportional fairness constraints. It is the first time that a closedform expression for end-to-end throughput of a proportional fair relaying, cooperative multi-hop path is presented.

\section{REFERENCES}

[1] A. Sendonaris, E. Erkip, and B. Aazhang, "User cooperation diversity. part i. system description," IEEE Trans. Commun., vol. 51, no. 11, pp. 1927-1938, November 2003.

[2] — "User cooperation diversity. part ii. implementation aspects and performance analysis," IEEE Trans. Commun., vol. 51, no. 11, pp. 1939 1948, November 2003.

[3] J. N. Laneman, D. N. C. Tse, and G. W. Wornell, "Cooperative diversity in wireless networks: Efficient protocols and outage behavior," IEEE Trans. Inf. Theory, vol. 50, no. 12, pp. 3062-3080, 2004.

[4] L. Dai, B. Gui, and L. J. Cimini, "Selective relaying in ofdm multihop cooperative networks," in Proc. IEEE Wireless Communications and Networking Conference WCNC 2007, March 2007, pp. 963-968.

[5] A. Bletsas, A. Khisti, D. P. Reed, and A. Lippman, "A simple cooperative diversity method based on network path selection," IEEE J. Sel. Areas Commun., vol. 24, no. 3, pp. 659-672, March 2006.

[6] F. Kelly, "Charging and rate control for elastic traffic," European Transactions on Telecommunications, vol. 8, pp. 33-37, February 1997.

[7] S. Borst, "User-level performance of channel-aware scheduling algorithms in wireless data networks," in Proc. INFOCOM 2003. TwentySecond Annual Joint Conference of the IEEE Computer and Communications Societies. IEEE, vol. 1, March 2003, pp. 321-331.

[8] E. Liu and K. K. Leung, "Fair resource allocation under rayleigh and/or rician fading environments," in Proc. IEEE 19th International Symposium on Personal, Indoor and Mobile Radio Communications PIMRC 2008, September 2008, pp. 1-5.

[9] — " "On proportional fair scheduling in multi-antenna wireless mesh networks-theoretical analysis," in Proc. IEEE Global Telecommunications Conference IEEE GLOBECOM 2008, November/December 2008, pp. $1-5$.

[10] P. J. Smith and M. Shafi, "On a gaussian approximation to the capacity of wireless mimo systems," in Proc. IEEE International Conference on Communications ICC 2002, vol. 1, April 2002, pp. 406-410.

[11] P. J. Smith, S. Roy, and M. Shafi, "Capacity of mimo systems with semicorrelated flat fading," IEEE Trans. Inf. Theory, vol. 49, no. 10, pp. 2781-2788, October 2003

[12] Y. Gao, D.-M. Chiu, and J. C. Lui, "Determining the end-to-end throughput capacity in multi-hop networks: methodology and applications," in Proc. ACM SIGMETRICS/IFIP Performance 2006, vol. 34, no. 1, Saint Malo, France, June 2006, pp. 39-50. 